\title{
Generation of a severe convective ionospheric storm under stable Rayleigh-Taylor conditions: triggering by meteors?
}

\author{
M. C. Kelley and R. R. Ilma \\ School of Electrical and Computer Engineering, Cornell University, Ithaca, NY 14853, USA \\ Correspondence to: M. C. Kelley (mck13@cornell.edu)
}

Received: 25 September 2015 - Revised: 27 December 2015 - Accepted: 5 January 2016 - Published: 3 February 2016

\begin{abstract}
Here we report on four events detected using the Jicamarca Radio Observatory (JRO) over an 18-year period, in which huge convective ionospheric storms (CISs) occur in a stable ionosphere. We argue that these rare events could be initiated by meteor-induced electric fields. The meteorinduced electric fields map to the bottomside of the $\mathrm{F}$ region, causing radar echoes and a localized CIS. If and when a localized disturbance reaches $500 \mathrm{~km}$, we argue that it becomes two-dimensionally turbulent and cascades structure to both large and small scales. This leads to long-lasting structure and, almost certainly, to scintillations over a huge range of latitudes some $\pm 15^{\circ}$ wide and to $3 \mathrm{~m}$ irregularities, which backscatter the VHF radar waves. These structures located at high altitudes are supported by vortices shed by the upwelling bubble in a vortex street.
\end{abstract}

Keywords. Ionosphere (equatorial ionosphere; ionospheric irregularities) - radio science (ionospheric physics)

\section{Introduction}

Many features of convective ionospheric storms (CISs), a.k.a. equatorial spread $\mathrm{F}(\mathrm{ESF})$, are understood now after 90 years of studies (see review by Kelley et al., 2011). A CIS manifests itself as vertically elongated wedges of depleted plasma (bubbles) that drift upward from beneath the bottomside $\mathrm{F}$ region to the plasmasphere. Most of the CIS-related phenomena have been related to the ionospheric interchange instability (Haerendel, 1973). Here we examine data, some of them collected almost 20 years ago, that are so baffling they have not even been published. The CIS events presented in this study occurred in the bottomside of the $\mathrm{F}$ region with a very low growth rate. These rare events rapidly develop into a narrow plume reaching $\mathrm{F}$ region topside altitudes. We provide a possible explanation associated with the seeding of ESF by meteor-induced electric fields. Such fields have been invoked to explain long-duration radar echoes, attributing them to the Farley E region instability (Farley, 1963) driven by these fields. The Farley instability is excited if the component of the electron-ion relative drift in the direction of propagation of the irregularities $\left(\mathbf{k} \cdot \mathbf{V}_{\mathrm{D}}\right)$ exceeds the ion acoustic speed $\left(C_{\mathrm{s}}\right)$. Extensive details on CISs and Farley instability can be found in Kelley (2009). There is ample evidence that electric fields driven by meteors exist, and here we argue that they may also induce CISs in the F region.

\section{Data Presentation}

Figures 1 and 2 show the two initial examples we found many years ago but never understood. According to the World Data Center (WDC) for Geomagnetism, Kyoto, the magnetic activity during these two events was low $(\mathrm{Kp}=2)$. More typical events are presented in Kelley (2009). The dark areas show regions of backscatter from $3 \mathrm{~m}$ irregularities detected by the large Jicamarca radar in Peru $\left(11.95^{\circ} \mathrm{S}\right.$, $76.87^{\circ} \mathrm{W} ; 0.6^{\circ} \mathrm{N}$ dip latitude, $1.0^{\circ}$, dip angle). The system works like a slit camera looking straight up and the resulting view looks south at the ionospheric equatorial plane with east to the left and west to the right (see Fig. 4). For a rigid structure moving at the typical value of $100 \mathrm{~m} \mathrm{~s}^{-1}$, the scales are designed such that the shape resembles a real photo.

These data have many unusual aspects. When these impressive plumes erupt, the ionosphere is very low and descending in both cases, meaning that the equatorial zone is nearly stable, or at least has a very low growth rate, for CISs driven locally. In Fig. 2, essentially no initial irregularities exist prior to the eruption, that is, no local seeding is present. The gray areas in the plot most likely are noise. The initial size of the plumes, corresponding to their 5 min duration and 


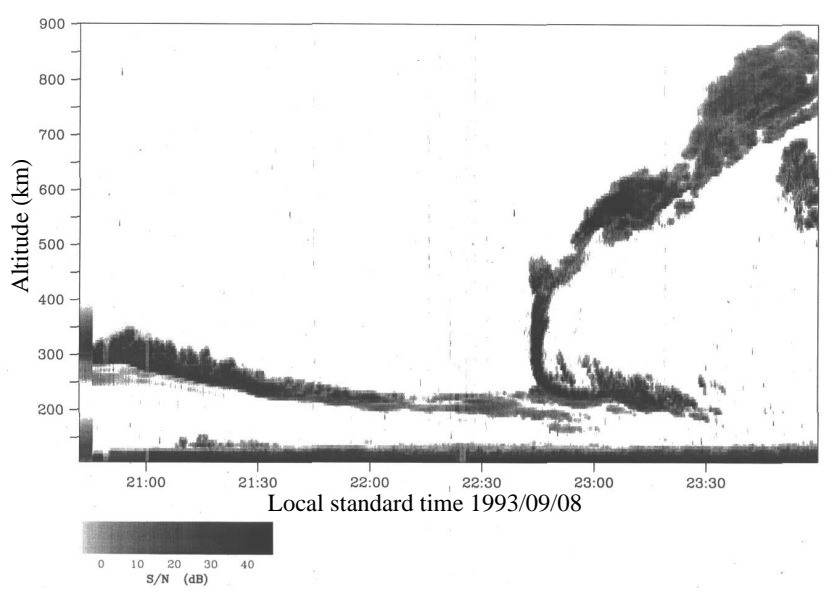

Figure 1. Range-time-intensity plot of spread-F irregularities on 8 September 1993. The C-shaped structure appears at 22:45 LT. The level of magnetic activity during the event is low $(\mathrm{Kp}=2)$.

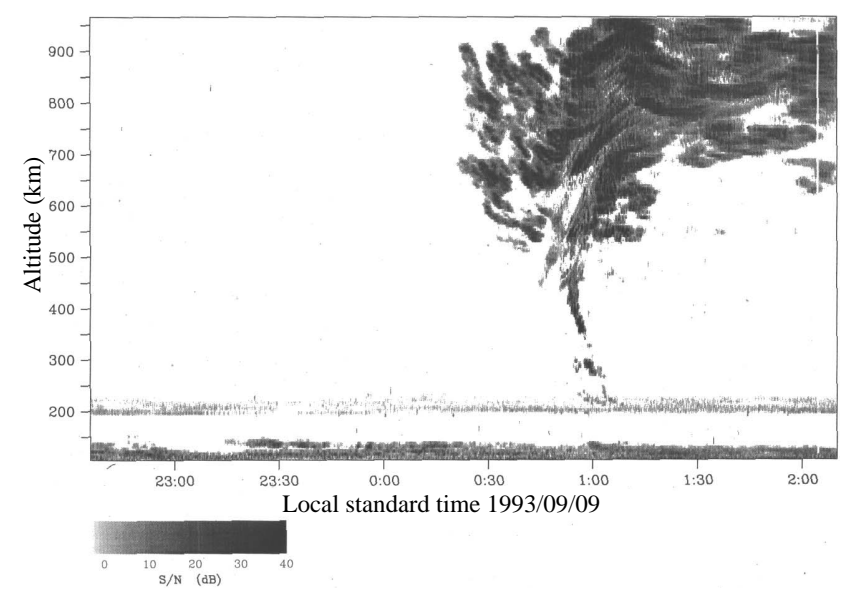

Figure 2. The same as Fig. 1 but on 9 September 1993. This event occurred during low magnetic activity $(\mathrm{Kp}=2)$.

to a spatial structure drifting eastward at $100 \mathrm{~m} \mathrm{~s}^{-1}$, is $30 \mathrm{~km}$. The final state in the two cases is similar in some ways and very different in others.

In Fig. 1, the narrow structure rises straight up and expands somewhat above $500 \mathrm{~km}$ and then seems to explode to $900 \mathrm{~km}$, the top of the data limit. Notice that, although there is turbulence in the plume, it is less extensive than in Fig. 2. Woodman and La Hoz (1976) called the structure in Fig. 1 a C-shaped structure. There are at least two explanations for the C-shaped feature. Woodman and La Hoz (1976) argued that, as a bubble or density depletion rising in the presence of a downward background electric field, it polarizes such that the internal electric field is upward. This means the bubble plasma moves slower than the background and, since it is turbulent, the radar signal follows it. Thus, the plume signal comes to the radar beam as a function of height forming the top of the $\mathrm{C}$. The bottom connects to the bottomside layer

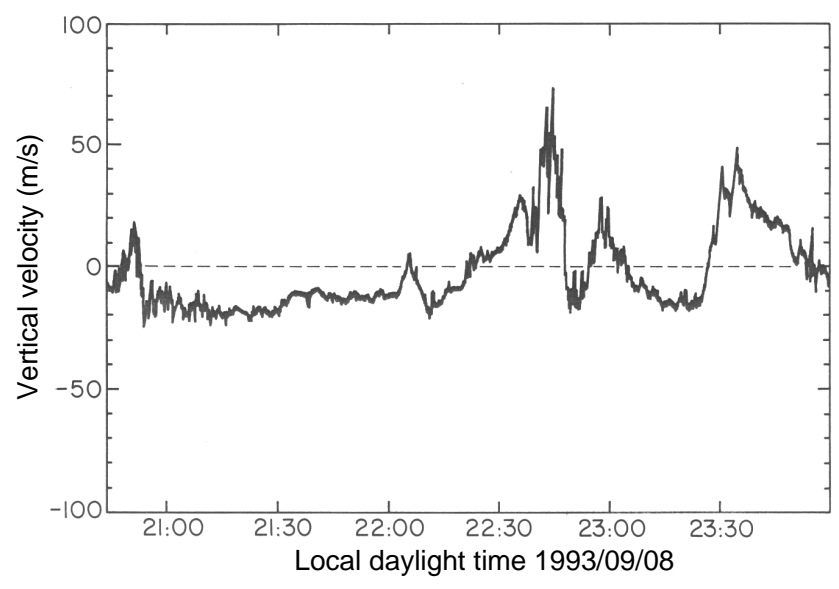

Figure 3. Vertical velocity of plasma irregularities corresponding to Fig. 1.

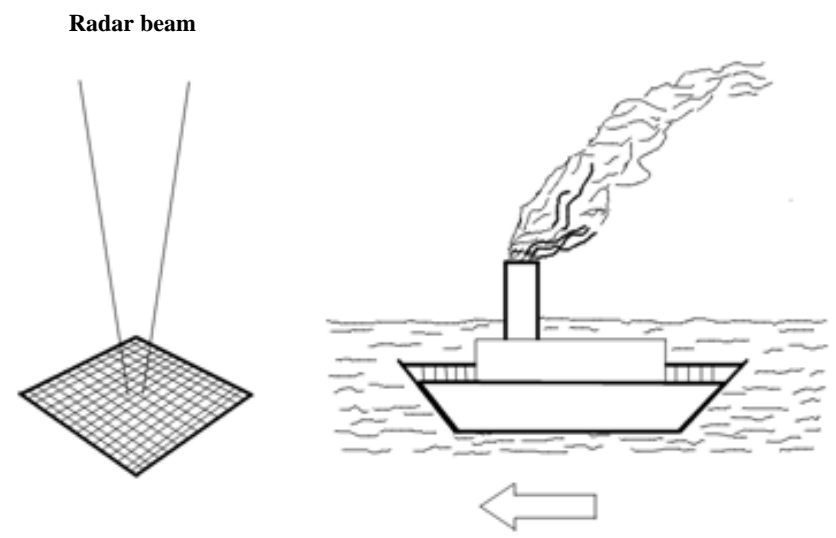

Figure 4. Cartoon showing how a slit camera would view the plume from a boat moving east into a velocity shear.

to finish the $\mathrm{C}$. The second explanation is that the downward electric field component is partially shorted out by the offequatorial E region (Zalesak et al., 1982). The bottom portion of the $\mathrm{C}$ is completed by a westward flow in the region below the F peak (Fejer et al., 1975) in either case. Figure 2 exhibits a small $\mathrm{C}$, almost no bottomside echoes, and the initial plume width is even smaller than in Fig. 1. However, above $500 \mathrm{~km}$, it erupts into a huge structure with a turbulent volume of over $4 \times 10^{8}$ cubic kilometers, where we have used the length of the magnetic field line for the third dimension. We only have line-of-sight Doppler information on 8 September, as shown in Fig. 3.

Some of the echoes in Fig. 3 are from the irregularities, not from incoherent scatter, but the drifts before and after the plume agree with the motion of the layer. Every aspect of the event indicates that the generalized Rayleigh-Taylor instability (GRTI) was stable before the events. The electric field was westward, so the $\boldsymbol{E} \times \boldsymbol{B}$ instability was stable on the bottomside. Due to the low altitude, the GRTI growth rate was small. The Doppler velocity agrees with the motion of the 

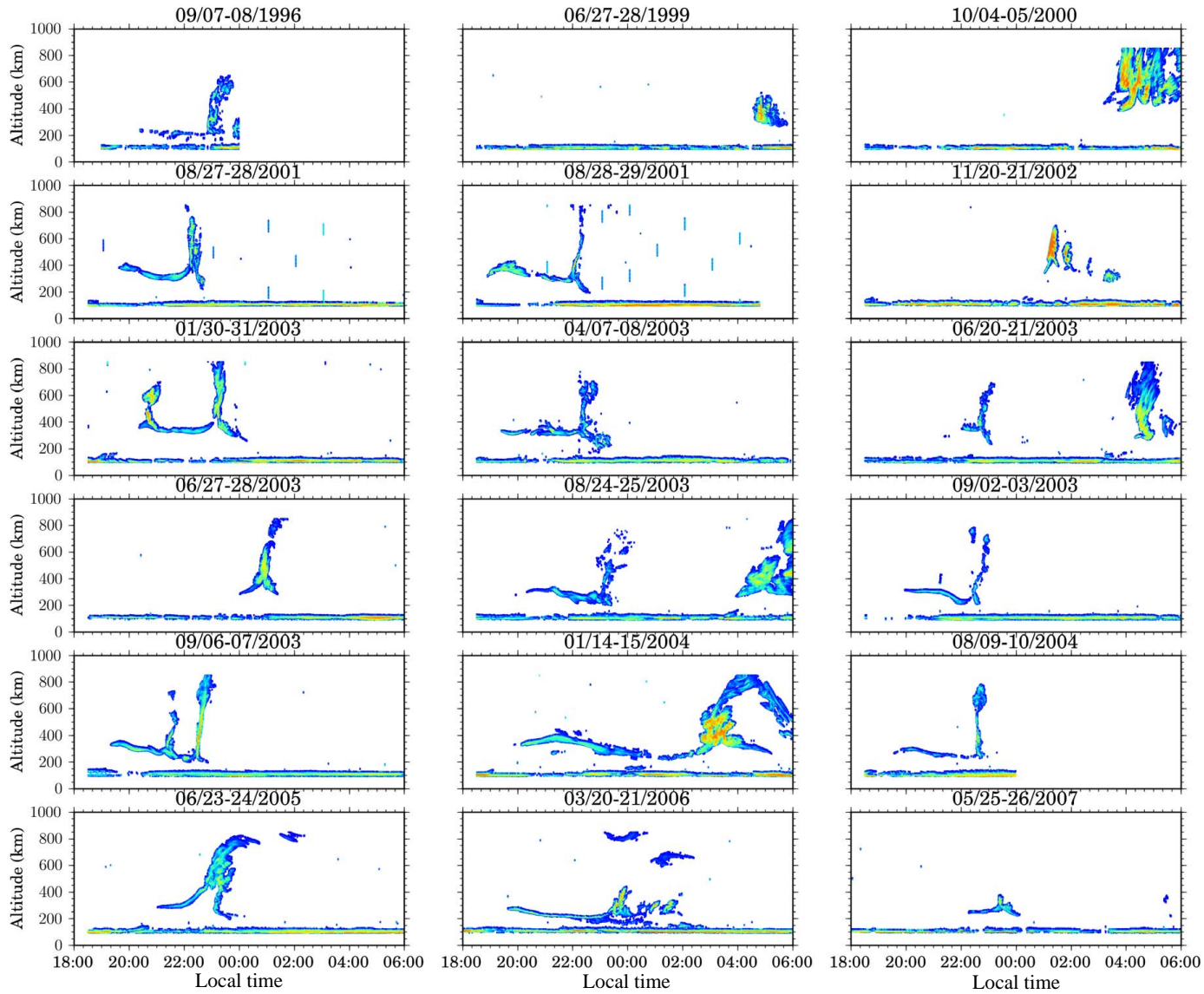

Figure 5. Eighteen range-time-intensity plots of spread-F events. Using the JULIA radar, these are similar-looking events.

echoing region every time and indicates that a large perturbation electric field $\left(1 \mathrm{mV} \mathrm{m}^{-1}\right)$ developed quickly at 22:40 LT. We cannot determine the solar wind electric field at this time since there was no upstream satellite, but a temporal pulse of penetrating electric field, rather than an unstable (preexisting) feature, would not have time to create the plume. For example, at $50 \mathrm{~m} \mathrm{~s}^{-1}$ the plume would take over $1 \mathrm{~h}$ to rise to $450 \mathrm{~km}$, not less than $5 \mathrm{~min}$ as indicated. Thus, the structure must have existed prior to 22:40 LT and just drifted over the site with its internal perturbation field inside. This reminds us of a snapshot of a boat, with its smokestack billowing, traveling to the left with the plume entering a region of velocity shear, as shown in Fig. 4.

Eighteen more or less similar events are shown in Fig. 5. The two events that we feel are very similar to those in Figs. 1 and 2 are reproduced in Fig. 6. Our criterion of similarity is based on their low initial altitude and the lack of any uplift prior to the event. The data selected was obtained with the JULIA radar (Jicamarca Unattended Long-term investigation of the Ionosphere and Atmosphere) (Hysell et al., 1997; Hysell and Burcham, 1998). Note that the JULIA system transmits at a $10 \mathrm{db}$ smaller power and hence the plumes in Figs. 1 and 2 would look smaller to JULIA. Other possibilities we

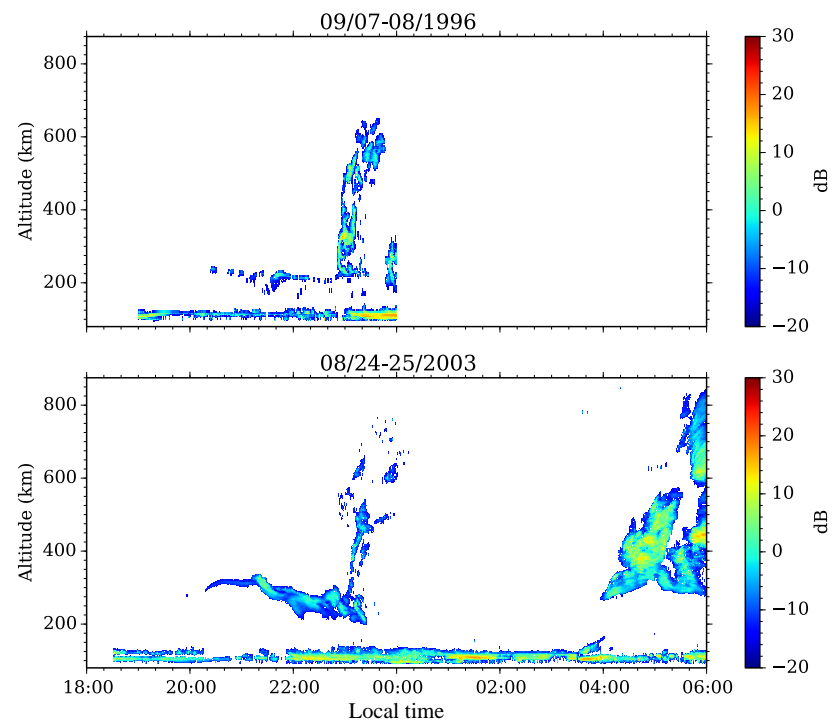

Figure 6. Two of the Fig. 5 events are shown, which we believe are similar to those in Figs. 1 and 2. These events occurred under moderate magnetic activity. The Kp indices are 3 (top) and 5 (bottom). 
have rejected are based on the plumes' size at the base, the launching of an "apogee" plume if the layer reaches a high enough altitude, or plumes clearly seeded by either a gravity wave or a shear instability. The fact that we only found four examples between 1993 and 2007 proves that these events are indeed rare.

\section{Discussion}

The events are so rare that the source must also be very rare. The source must create a large electric field at $200-250 \mathrm{~km}$ and be localized in space and time. Our only candidate is a large meteor.

We know that meteors do create electric fields; the $\mathrm{Ji}$ camarca radar has detected two-stream waves (Chapin and Kudeki, 1994; Oppenheim and Otani, 1996) due to meteors. The cause is an ambipolar electric field due to the localized trail of atoms and plasma as the meteor ablates, vaporizes, and ionizes (Oppenheim et al., 2003).

Kelley and Collin (2016) have presented a new model for the electrodynamics of meteor entry into the mesospherelower thermosphere and the $\mathrm{D}$ region of the ionosphere. Their purpose was to explain how humans are able to hear meteors at the same time they see them. Using the geometry below they suggested that the current in the E region launches an audio frequency radio wave, which couples on metallic objects to a sound wave by the process of electrophonics. The idea is illustrated here in Fig. 7.

In meteor radar studies there is a phenomenon known as a head echo in which a dense plasma, as high as $10^{16} \mathrm{~m}^{-3}$, is formed and follows the meteor itself. Curiously this plasma disappears a few meters behind the meteor and is hence called a coma. Since there are known to be electrons around the meteor head due to the radar head echo, there must also be ions. The ions are borne with the same velocity as the meteor and must travel with it in the D region where they are not magnetized. However, the electrons are magnetized and cannot follow any component of the ion motion across the magnetic field, $\boldsymbol{B}$, unless an electric field builds up to drag the electrons along. To accomplish this requires a large electric field since the electrons are magnetized. To keep $n_{\mathrm{i}}=n_{\mathrm{e}}$, an electric field builds up such that the ion current equals the electron Pedersen current (the component parallel to $\boldsymbol{E}$ in the anisotropic ionospheric current). Using the electron conductivity in the D region (Kelley, 2009),

$E_{x}=\frac{e n V_{\mathrm{m}}}{\sigma_{\mathrm{eP}}}$,

where $V_{\mathrm{m}}$ is the meteor velocity, $n$ is the initial ambient plasma density, and $\sigma_{\mathrm{eP}}$ is the electron Pedersen conductivity, which is proportional to the density. Substituting yields $E_{x}=10 \mathrm{~V} \mathrm{~m}^{-1}$. As discussed by Kelley and Collin (2016), this is greater than the critical velocity for neutral atmospheric discharge that is known to occur over thunderstorms,

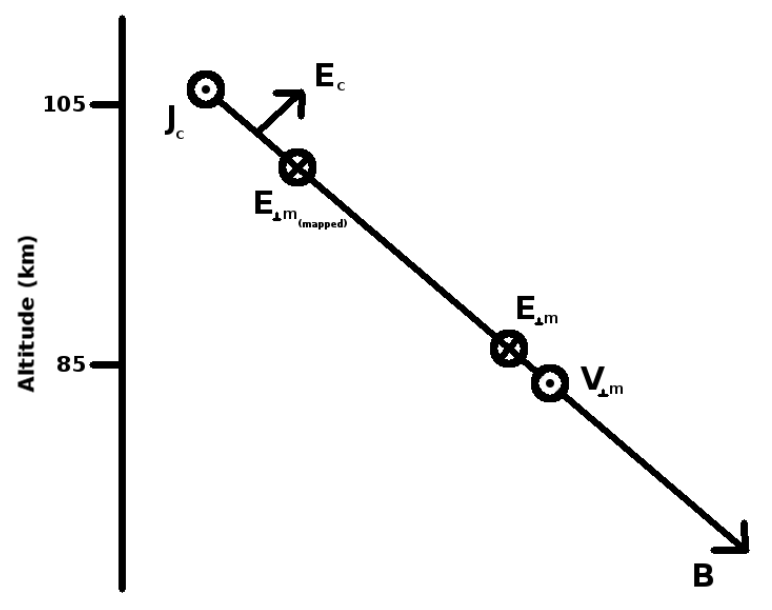

Figure 7. Geometry of the model. Here we take the optimum case for a meteor velocity perpendicular to $\boldsymbol{B}$. The electric field generated opposite of the meteor velocity maps up to the E region since the conductivity along $\boldsymbol{B}$ is very high and is the source of the electromagnetic radiation needed for electrophonics. The $E_{\mathrm{m}}$ field is the component we discuss below and which travels to the bottom of the F layer as a whistler pulse.

creating the phenomenon of sprites. We argue that the E field saturates at the critical value for discharge, which, at the meteor ablation height, is about $2 \mathrm{~V} \mathrm{~m}^{-1}$.

The region of discharge is behind the meteor and only as large as the size of the electric field. We estimate this as follows. The separation of ions and electrons can only be on the order of a few Debye lengths $\left(\lambda_{D}\right)$. In the initial background ionosphere the Debye length is $\lambda_{\mathrm{D}}=V_{\mathrm{e}} / f_{\mathrm{p}}$, where $V_{\mathrm{e}}$ is the electron thermal speed (about $10^{6} \mathrm{~m} \mathrm{~s}^{-1}$ ) and $f_{\mathrm{p}}$ is the plasma frequency (about $10^{5} \mathrm{~Hz}$ ). Thus, initially $\lambda_{\mathrm{D}}$ is about $10 \mathrm{~m}$ and the electric field extends $20-30 \mathrm{~m}$. The physics after this time is very complex as the electrons heat and the plasma density increases. The two effects tend to cancel in the estimate of the electric field length.

This electric field is launched as a millisecond-long whistler mode wave pulse along the field line, arriving at the base of the $\mathrm{F}$ region almost unattenuated. Within $1 \mathrm{~s}$ the entire length of the meteor track $(10-40 \mathrm{~km})$ has launched this large field, which when it reaches the base of the F layer lifts the plasma at a huge velocity, some $80 \mathrm{~km} \mathrm{~s}^{-1}$. This clearly punches through to the topside, creating a huge disturbance,ff7 and certainly can cause the observed phenomena.

Ott (1978) showed that a $30 \mathrm{~km}$ bubble will begin to behave in a two-dimensionally turbulent manner (Kraichnan, 1967) at a height of about $500 \mathrm{~km}$. When this occurs, the turbulent region will undergo an inverse cascade to larger and larger sizes, explaining the huge volume of radar scatter in these events. The huge path lengths also increase the scintillation of radio waves. An event like the one in Fig. 1 will cause an S4 of nearly 1 at GPS frequencies. 
The transition between the collisional and collisionless Rayleigh-Taylor instability occurs at a height where the ionneutral collision frequency is such that

$v_{\text {in }}=\frac{16 U}{R}$,

where $R$ is the two-dimensional bubble radius and $U$ is the bubble velocity (Ott, 1978). In this case, the growth rate is

$\gamma=\frac{g}{\Omega_{\mathrm{i}} L}$

where $\Omega_{\mathrm{i}}$ is the ion gyro frequency and $g$ is the gravitational constant. Initially in the collisionless case, the wedge (bubble) accelerates at $-g$. The terminal velocity in this case generates waves at marginal stability. Since this is all occurring perpendicular to $\boldsymbol{B}$, the instability is the Farley two stream, in which the ion current, driven by gravity, exceeds the sound speed, $C_{\mathrm{s}}$. In the resulting steady state, the charge that tends to build up inside the bubble as it tries to accelerate is shed back into the medium by vortices that occur in a vortex street behind the bubble (Ott, 1978). A vortex in a two-dimensional plasma is equivalent to a line charge, positive east of the bubble and negative west of it. Evidence for such vortices can be seen in Fig. 1. The terminal velocity is thus about $1 \mathrm{~km} \mathrm{~s}^{-1}$, a typical upwelling velocity at high altitudes (Hysell et al., 2005). The vortices must be long-lasting to support $3 \mathrm{~m}$ echoes for hours. This can only be maintained by the forward cascade of enstrophy down to meter scales, as occurs in two-dimensional turbulence (Kraichnan, 1967; Earle and Kelley, 1993). Furthermore, such circulation in a high-density plasma will tend to put the neutral atmosphere in a motion similar to motions in the high-latitude polar circulation zone (Heppner and Miller, 1982). In this case, with a plasma density of $10^{6} \mathrm{~cm}^{-3}$, we predict that a neutral vortex will form in $0.5 \mathrm{~h}$. However, such features would be very difficult to detect using current techniques.

\section{Conclusions}

We believe the unusual events reported here are due to large meteors impacting the E region off the equator. The resulting electric fields map to the bottomside of the F region, causing radar echoes and a localized convective ionospheric storm. Once the localized disturbance reaches $500 \mathrm{~km}$, it becomes two-dimensionally turbulent and cascades structure to both large and small scales, leading to long-lasting scintillation over a huge range of latitudes some $\pm 15^{\circ}$ wide and to $3 \mathrm{~m}$ irregularities that backscatter the VHF radar waves. At high altitudes, the steady state is supported through vortices shed by the upwelling bubble in a vortex street. Meteor events have led to simulations of the Farley instability. This study might encourage meteor simulations of $\mathrm{F}$ region instabilities in the future.

\section{The Supplement related to this article is available online at doi:10.5194/angeo-34-165-2016-supplement.}

Acknowledgements. The work at Cornell was supported by the Atmospheric Science Section of the NSF and the School of Electrical and Computer Engineering at Cornell.

The topical editor, H. Kil, thanks two anonymous referees for help in evaluating this paper.

\section{References}

Chapin, E. and Kudeki, E.: Plasma wave excitation on meteor trails in the equatorial electrojet, Geophys. Res. Lett., 21, 2433-2436, doi:10.1029/94GL01705, 1994.

Earle, G. D. and Kelley, M. C.: Spectral evidence for stirring scales and two-dimensional turbulence in the auroral ionosphere, $\mathrm{J}$. Geophys. Res., 98, 11543, doi:10.1029/93JA00632, 1993.

Farley Jr., D. T.: A Plasma Instability Resulting in Field-Aligned Irregularities in the Ionosphere, J. Geophys. Res., 68, 6083-6097, doi:10.1029/JZ068i022p06083, 1963.

Fejer, B. G., Farley, D. T., Balsley, B. B., and Woodman, R. F.: Vertical structure of the VHF backscattering region in the equatorial electrojet and the gradient drift instability, J. Geophys. Res., 80, 1313-1324, doi:10.1029/JA080i010p01313, 1975.

Haerendel, G. R.: Theory of equatorial spread F, unpublished report, Max-Plank Institut fur Physik and Astrophysik, Garching, F.R.G., Germany, 1973.

Heppner, J. P. and Miller, M. L.: Thermospheric winds at high latitudes from chemical release observations, J. Geophys. Res., 87 1633-1647, doi:10.1029/JA087iA03p01633, 1982.

Hysell, D. L. and Burcham, J. D.: JULIA radar studies of equatorial spread F, J. Geophys. Res., 103, 29155-29168, doi:10.1029/98JA02655, 1998.

Hysell, D. L., Larsen, M. F., and Woodman, R. F.: JULIA radar studies of electric fields in the equatorial electrojet, Geophys. Res. Lett., 24, 1687-1690, doi:10.1029/97GL00373, 1997.

Hysell, D. L., Larsen, M. F., Swenson, C. M., Barjatya, A., Wheeler, T. F., Sarango, M. F., Woodman, R. F., and Chau, J. L.: Onset conditions for equatorial spread $\mathrm{F}$ determined during EQUIS II, Geophys. Res. Lett., 32, L24104, doi:10.1029/2005GL024743, 2005.

Kelley, M. C.: The Earth's Ionosphere: Plasma Physics and Electrodynamics, Second Edition, Academic Press, San Diego, CA, USA, 2009.

Kelley, M. C. and Collin, P.: On the generation of electrophonics by meteors, Geophys. Res. Lett., in press, 2016.

Kelley, M. C., Makela, J. J., de La Beaujardière, O., and Retterer, J.: Convective Ionospheric Storms: A review, Rev. Geophys., 49, RG2003, doi:10.1029/2010RG000340, 2011.

Kraichnan, R. H.: Inertial ranges in two-dimensional turbulence, Phys. Fluids, 10, 1417-1423, doi:10.1063/1.1762301, 1967.

Oppenheim, M. and Otani, N.: Spectral characteristics of the FarleyBuneman instability: Simulations versus observations, J. Geophys. Res., 101, 24573-24582, doi:10.1029/96JA02237, 1996. 
Oppenheim, M. M., Dyrud, L. P., and Ray, L.: Plasma instabilities in meteor trails: Linear theory, J. Geophys. Res., 108, 1063, doi:10.1029/2002JA009548, 2003.

Ott, E.: Theory of Rayleigh-Taylor bubbles in the equatorial ionosphere, J. Geophys. Res., 83, 2066-2070, doi:10.1029/JA083iA05p02066, 1978.

Woodman, R. F. and La Hoz, C.: Radar observations of F region equatorial irregularities, J. Geophys. Res., 81, 5447-5466, doi:10.1029/JA081i031p05447, 1976.
Zalesak, S. T., Ossakow, S. L., and Chaturvedi, P. K.: Nonlinear equatorial spread $\mathrm{F}$ - The effect of neutral winds and background Pedersen conductivity, J. Geophys. Res., 87, 151-166, doi:10.1029/JA087iA01p00151, 1982. 